\title{
Effective Fragment Potentials for Flexible Molecules: Transferability of Parameters and Amino Acid Database Supporting Information
}

Yongbin Kima, Yen Buia, Ruslan N. Tazhigulovb,c, Ksenia B. Bravayab, Lyudmila V. Slipchenko

a Department of Chemistry, Purdue University, West Lafayette, IN 47907, USA

${ }^{b}$ Department of Chemistry, Boston University, Boston, MA 02215, USA

c Division of Chemistry and Chemical Engineering, California Institute of Technology, Pasadena, CA 91125, USA
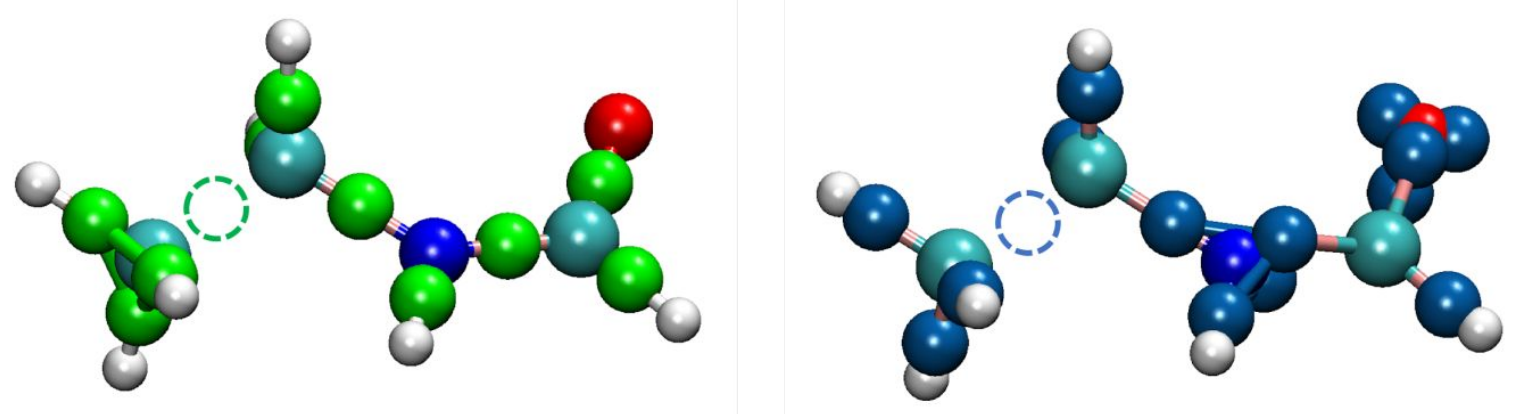

Fig. S1. Fragmentation into the backbone and side-chain groups on an example of the alanine residue. (Left) positions of bond mid-points (shown in green) and (right) positions of LMO centroids (shown in sapphire). Empty circles denote excluded parameters. 


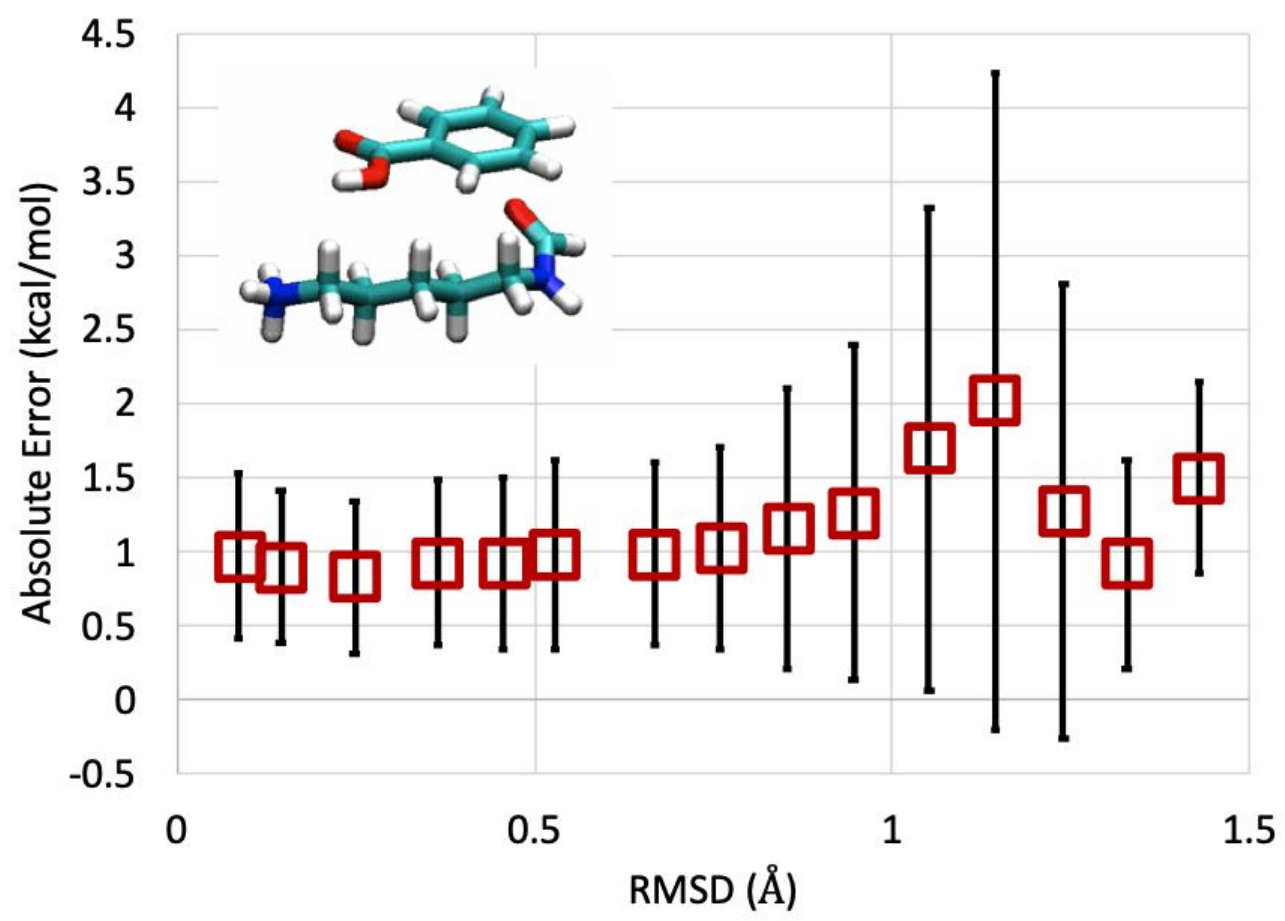

Fig. S2. Lysine-benzoic acid dimer at a separation $4 \AA$ between monomer centers of masses. Average absolute errors (red squares) and their standard deviations (black vertical lines), in $\mathrm{kcal} / \mathrm{mol}$, of the Flexible EFP scheme versus standard EFP, as a function of RMSD between lysine geometry in the dimer and lysine geometry in the EFPDB database. The data are averaged for 1000 geometries of lysine-benzoic acid dimer, each using one of the 54 database lysine EFP potentials (the total set contains 54000 data points). Benzoic acid monomer is kept rigid in all calculations; lysine geometries are extracted from the MD trajectory of cryptochrome. Absolute errors are averaged for RMSDs within $0.1 \AA$, i.e., for RMSD bins 0-0.1 $\AA, 0.1-0.2 \AA$, etc. The graph suggests that the accuracy of the Flexible EFP scheme is preserved for RMSD values below $0.7 \AA$. Interaction energies in the considered lysine-benzoic acid dimers vary between -10 and $+30 \mathrm{kcal} / \mathrm{mol}$, such that the observed average errors $<1 \mathrm{kcal} / \mathrm{mol}$ are consistent within a typical accuracy of EFP calculations. 

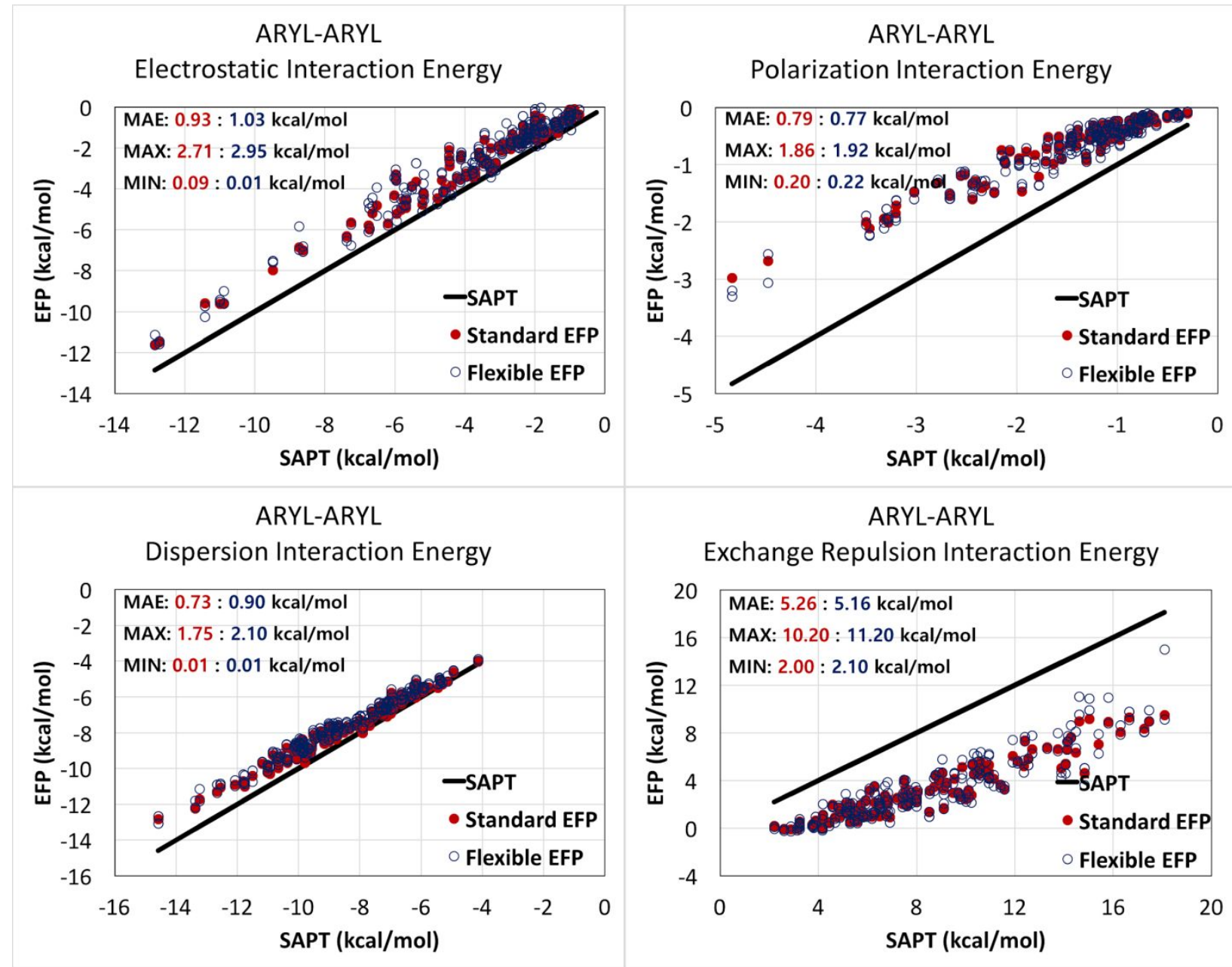

Fig. S3. Interaction energy components in aryl-aryl complexes computed with Standard EFP (red circles) and Flexible EFP (blue circles) against sSAPTO/jun-cc-pVDZ. 

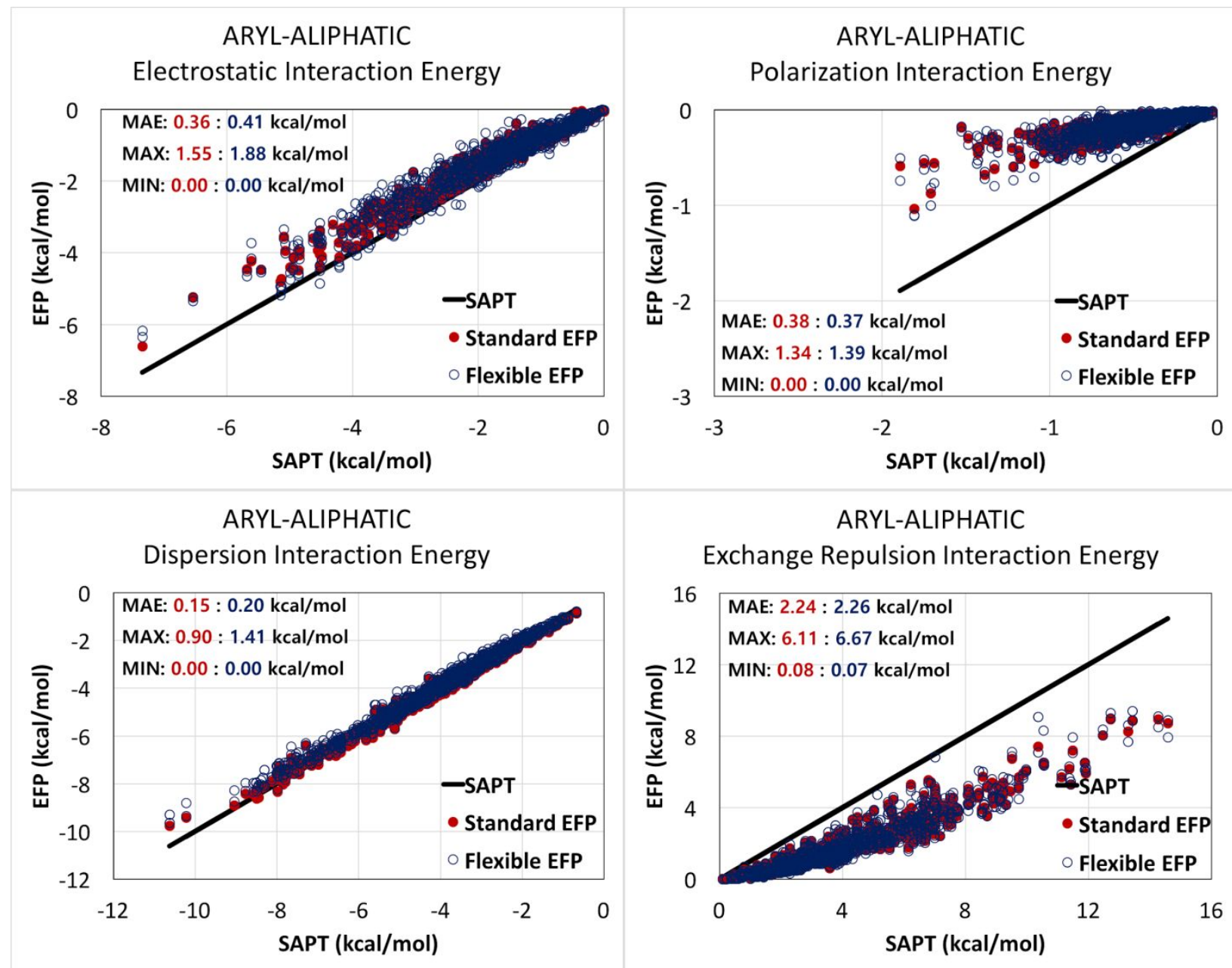

Fig. S4. Interaction energy components in aryl-aliphatic complexes computed with Standard EFP (red circles) and Flexible EFP (blue circles) against sSAPT0/jun-cc-pVDZ. 

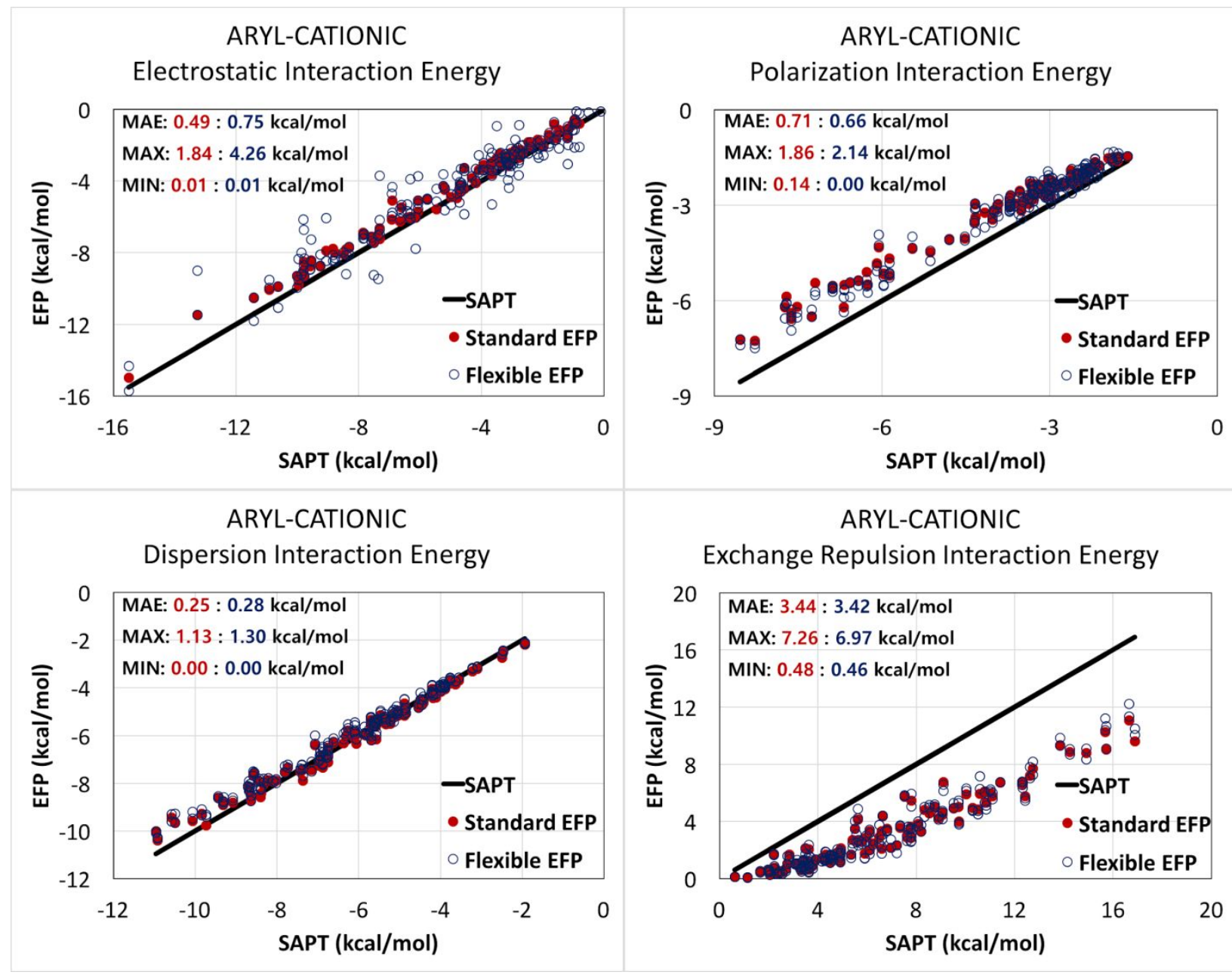

Fig. S5. Interaction energy components in aryl-cationic complexes computed with Standard EFP (red circles) and Flexible EFP (blue circles) against sSAPT0/jun-cc-pVDZ. 

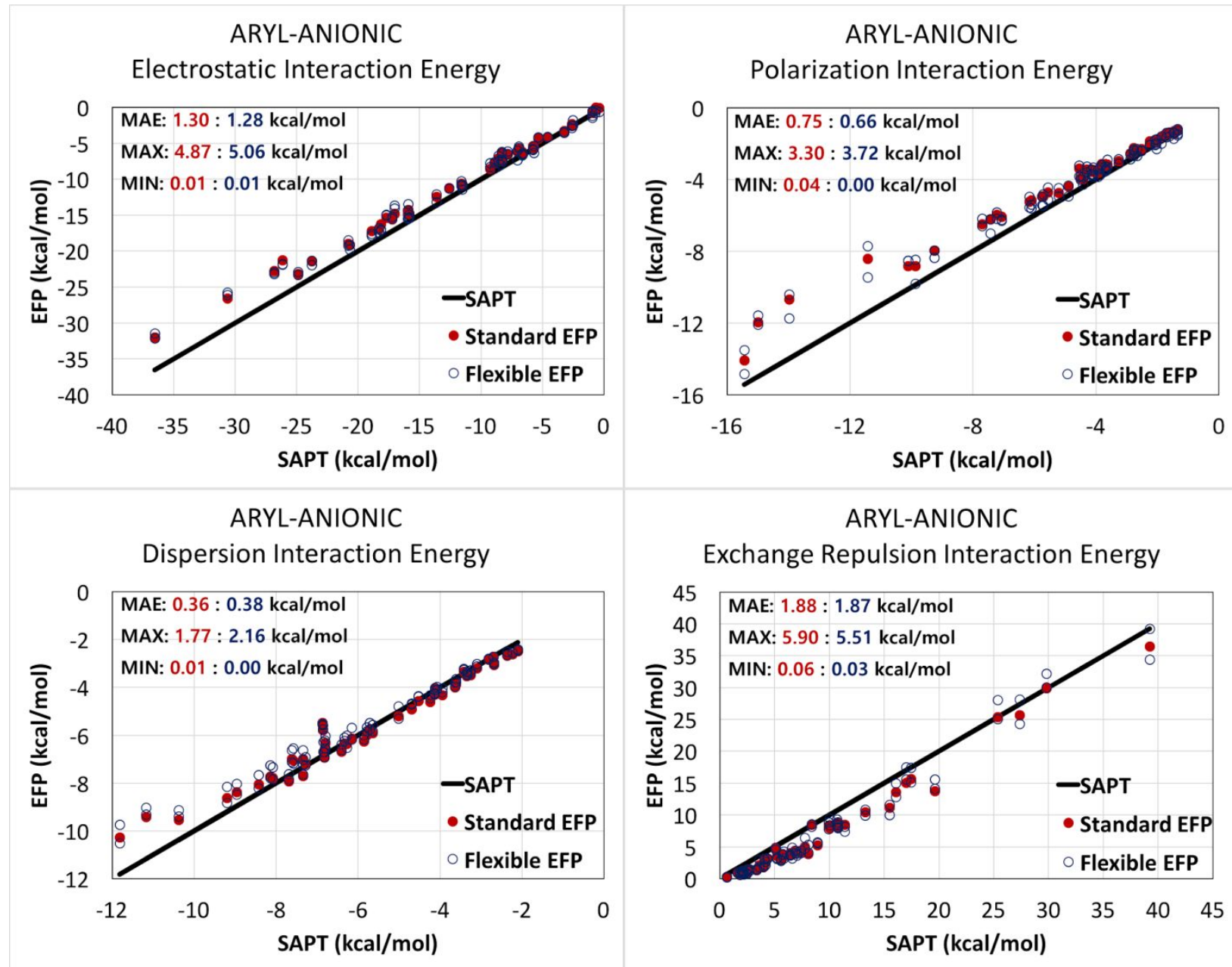

Fig. S6. Interaction energy components in aryl-anionic complexes computed with Standard EFP (red circles) and Flexible EFP (blue circles) against sSAPTO/jun-cc-pVDZ. 

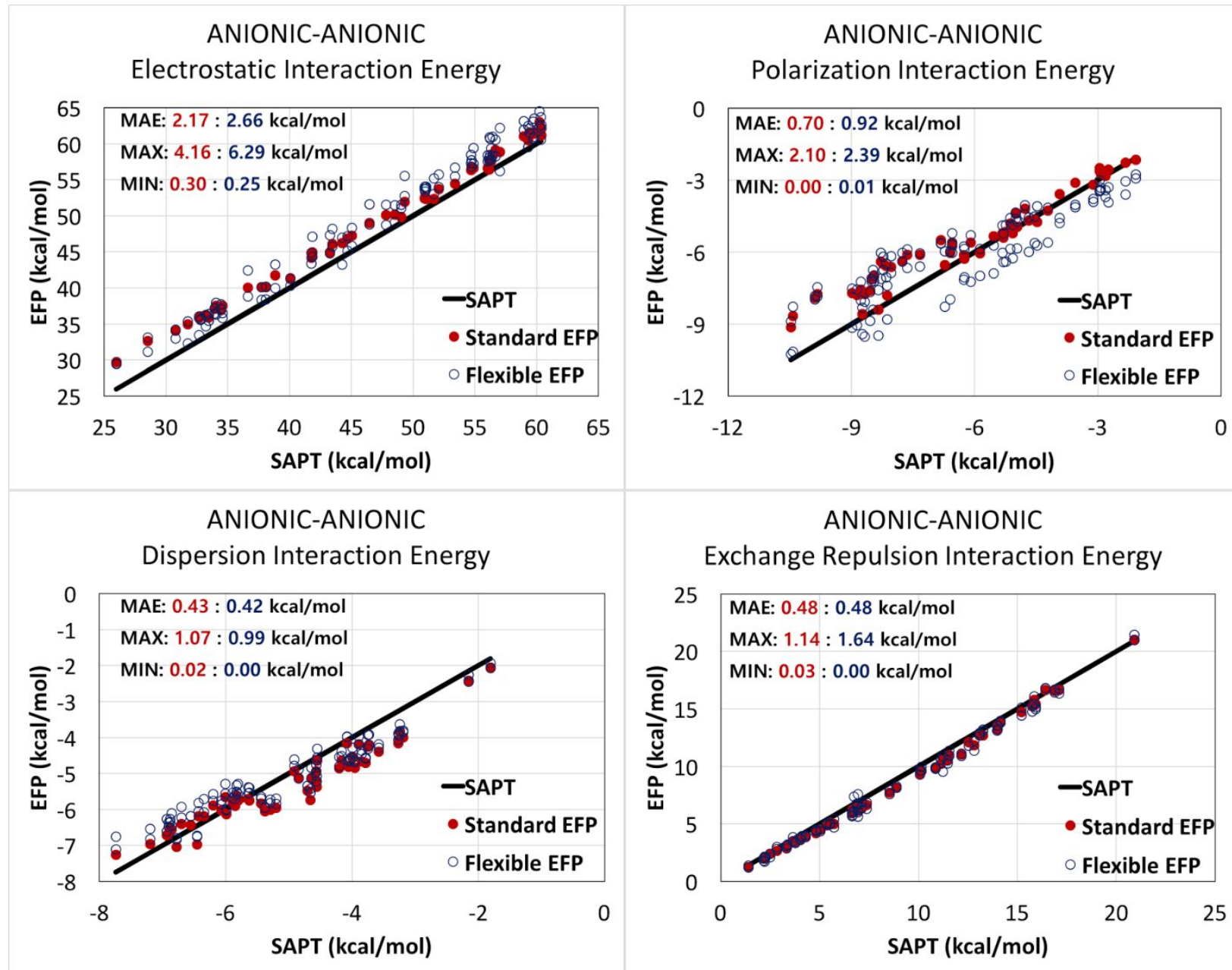

Fig. S7. Interaction energy components in anionic-anionic complexes computed with Standard EFP (red circles) and Flexible EFP (blue circles) against sSAPT0/jun-cc-pVDZ. 

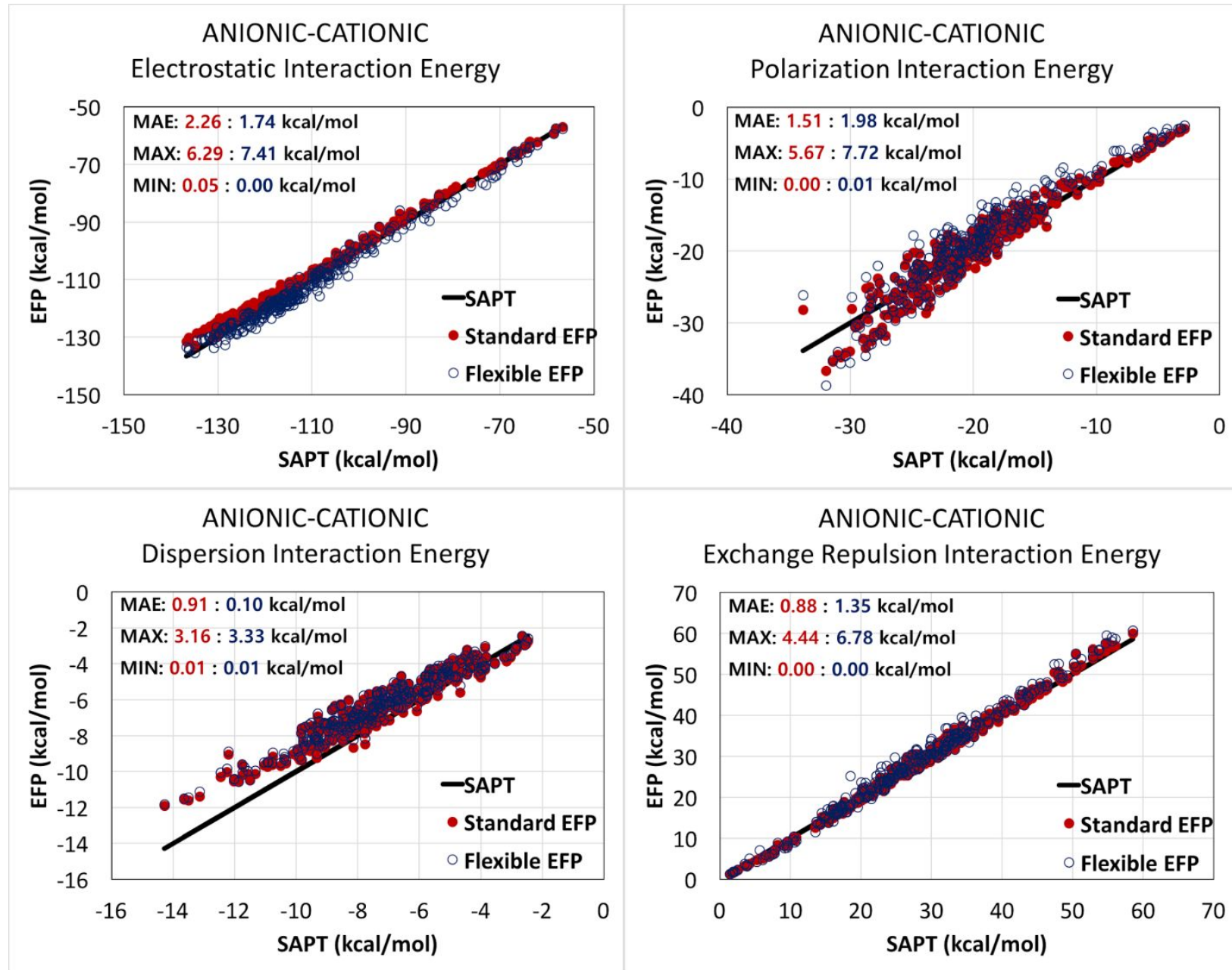

Fig. S8. Interaction energy components in anionic-cationic complexes computed with Standard EFP (red circles) and Flexible EFP (blue circles) against sSAPT0/jun-cc-pVDZ. 

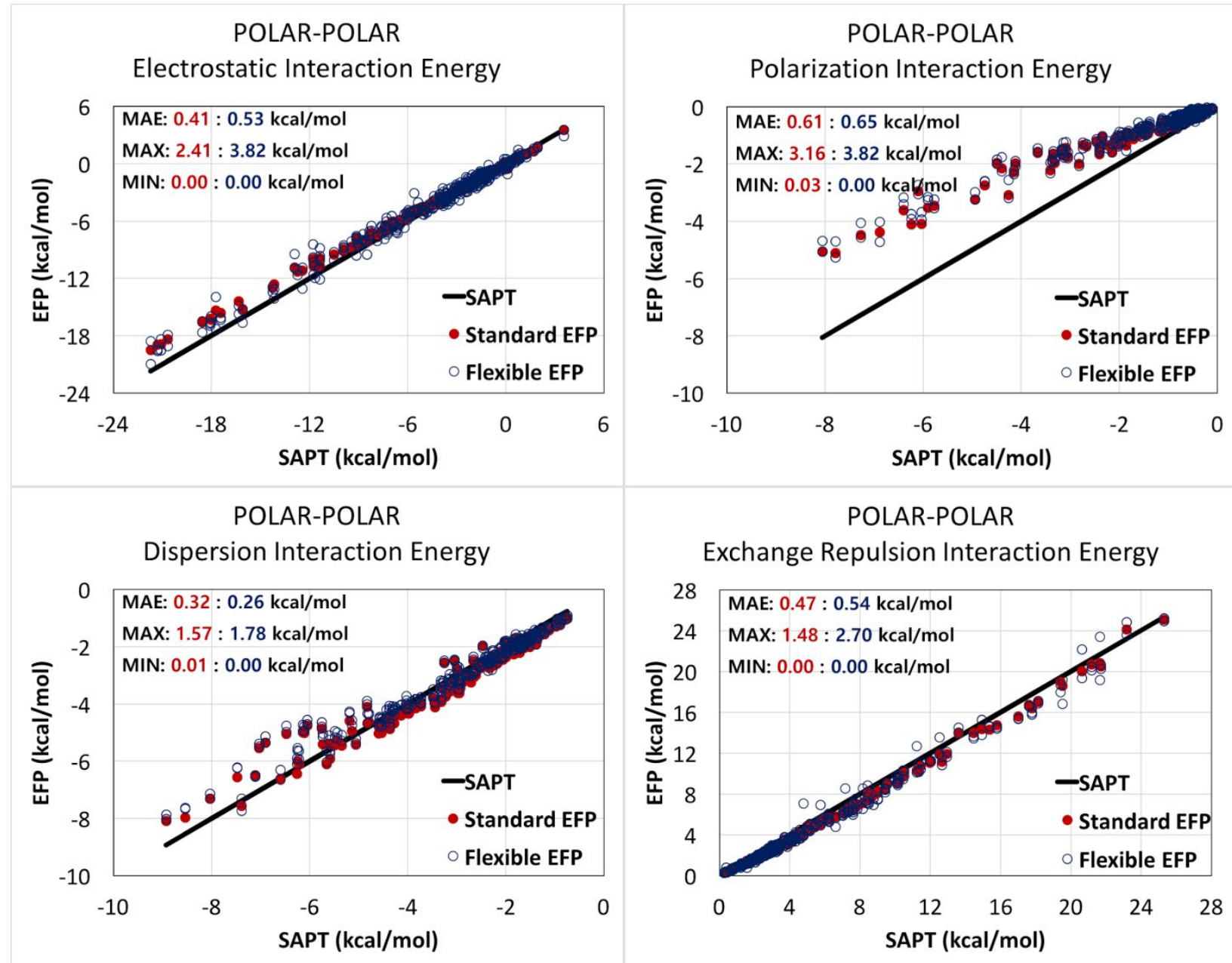

Fig. S9. Interaction energy components in polar-polar complexes computed with Standard EFP (red circles) and Flexible EFP (blue circles) against sSAPTO/jun-cc-pVDZ. 

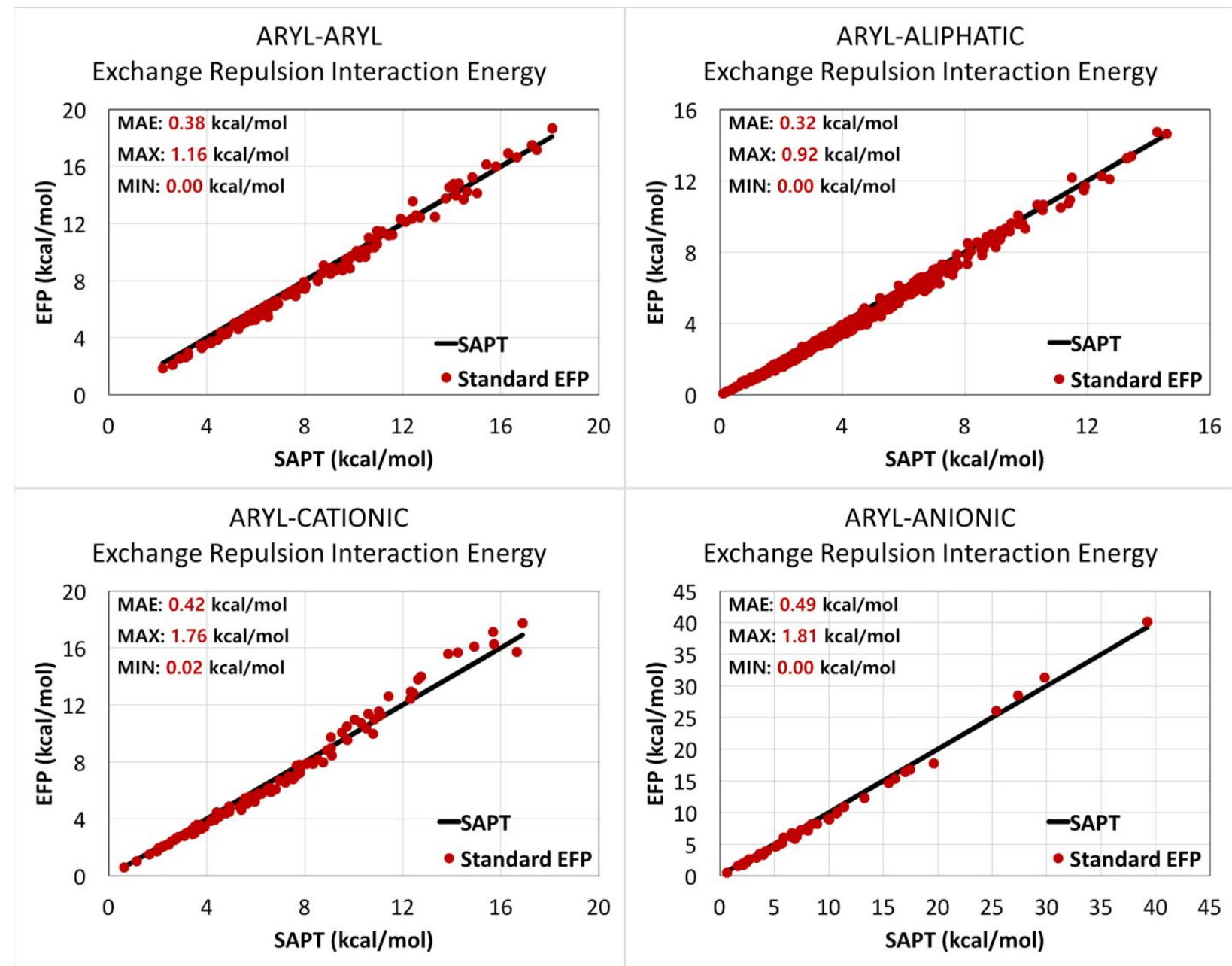

Fig. S10. Exchange-repulsion energies in aryl-containing complexes computed with Standard EFP (red circles) against sSAPT0/jun-cc-pVDZ. 

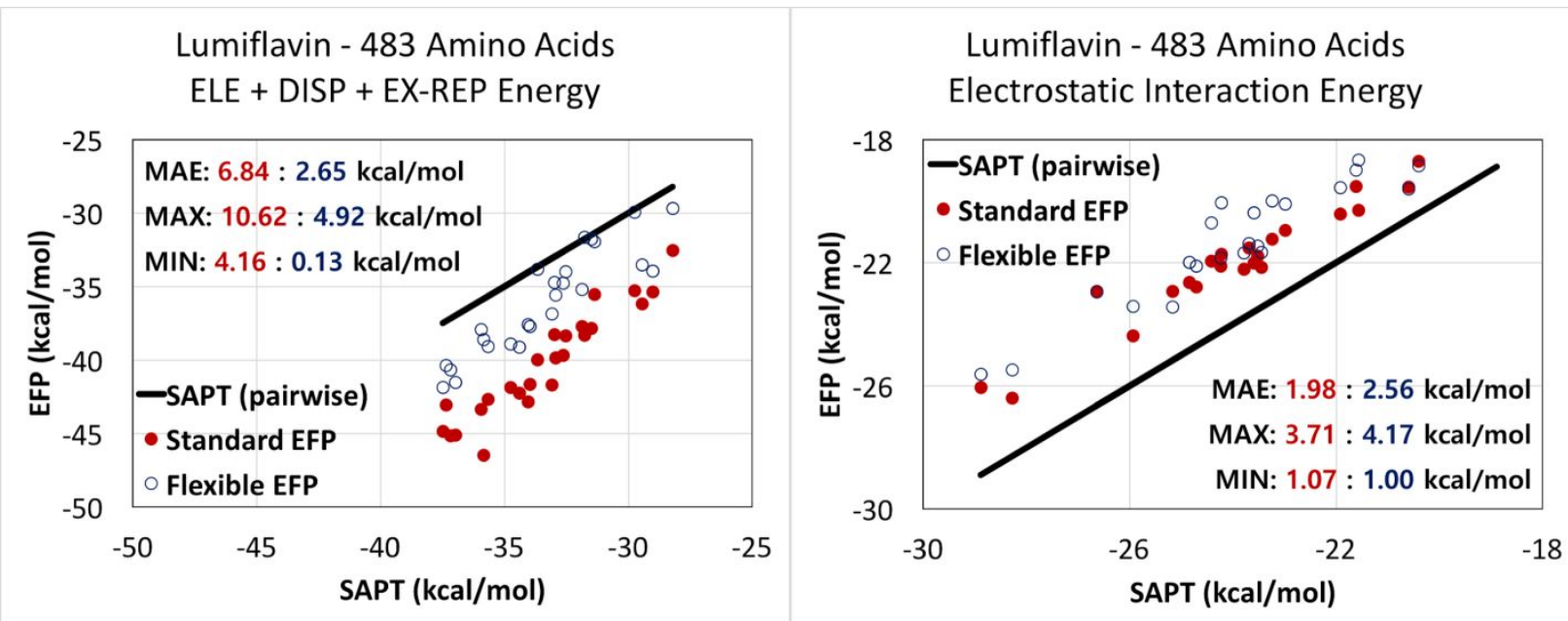

Lumiflavin - 483 Amino Acids

Dispersion Interaction Energy

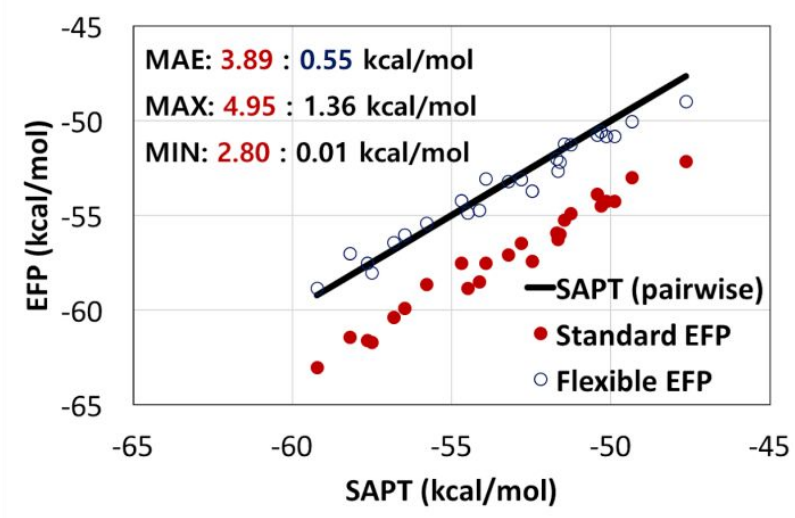

Lumiflavin - 483 Amino Acids

Exchange Repulsion Interaction Energy

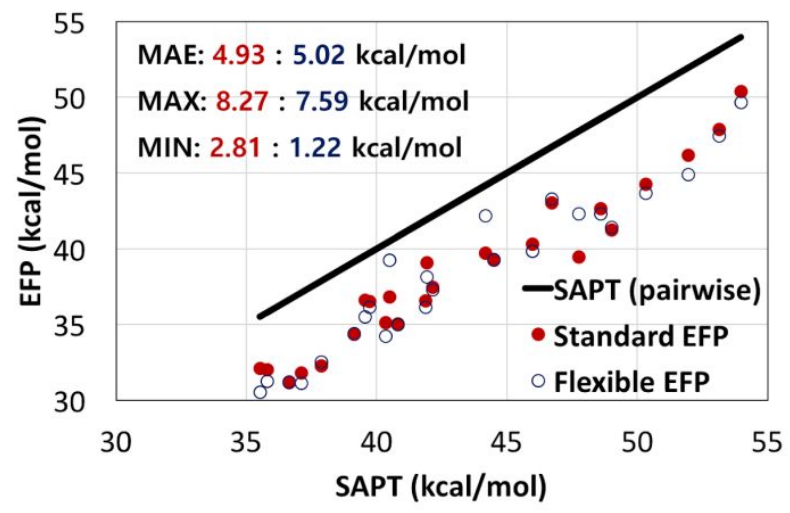

Fig. S11. Comparison of pairwise interaction energy components in the lumiflavin-protein system. Red circles: Standard EFP; blue circles: Flexible EFP. Comparison is done against sSAPT/jun-cc-pVDZ.
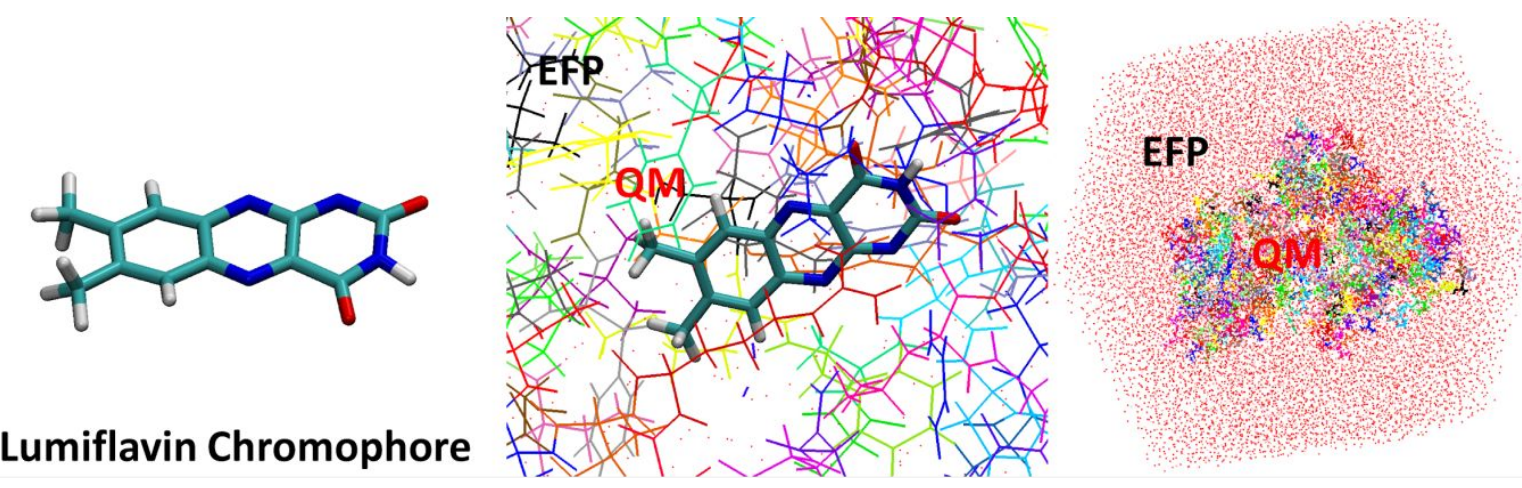

Fig. S12. Scheme of QM/EFP calculations of Cry1At protein. 
Table S1. VEA and VIE at each MD structure computed with the Standard and Flexible EFP schemes. OX and SEMI correspond to MD simulations for oxidized and semireduced forms of lumiflavin, respectively.

\begin{tabular}{|c|c|c|c|c|c|}
\hline \multirow{2}{*}{$\frac{\text { Ensemble }}{\text { OX }}$} & \multicolumn{2}{|c|}{ VEA, eV } & \multirow{2}{*}{$\begin{array}{c}\text { Ensemble } \\
\text { SEMI }\end{array}$} & \multicolumn{2}{|c|}{ VIE, eV } \\
\hline & Standard EFP & Flexible EFP & & Standard EFP & Flexible EFP \\
\hline snap_01 & 2.324 & 2.280 & snap_01 & 4.993 & 4.907 \\
\hline snap_02 & 2.703 & 2.642 & snap_02 & 4.766 & 4.764 \\
\hline snap_03 & 2.768 & 2.772 & snap_03 & 4.577 & 4.467 \\
\hline snap_04 & 2.390 & 2.383 & snap_04 & 4.882 & 4.835 \\
\hline snap_05 & 2.658 & 2.655 & snap_05 & 4.978 & 4.896 \\
\hline snap_06 & 2.431 & 2.372 & snap_06 & 4.861 & 4.840 \\
\hline snap_07 & 2.547 & 2.503 & snap_07 & 4.893 & 4.892 \\
\hline snap_08 & 2.573 & 2.568 & snap_08 & 5.083 & 5.036 \\
\hline snap_09 & 2.056 & 2.053 & snap_09 & 5.080 & 5.080 \\
\hline snap_10 & 2.630 & 2.603 & snap_10 & 4.858 & 4.784 \\
\hline snap_11 & 2.694 & 2.664 & snap_11 & 5.221 & 5.208 \\
\hline snap_12 & 2.480 & 2.426 & snap_12 & 4.752 & 4.750 \\
\hline snap_13 & 2.035 & 2.008 & snap_13 & 4.651 & 4.657 \\
\hline snap_14 & 2.876 & 2.902 & snap_14 & 5.189 & 5.171 \\
\hline snap_15 & 2.125 & 2.099 & snap_15 & 5.080 & 5.038 \\
\hline snap_16 & 2.359 & 2.365 & snap_16 & 5.112 & 5.030 \\
\hline snap_17 & 2.449 & 2.383 & snap_17 & 5.279 & 5.271 \\
\hline snap_18 & 2.702 & 2.573 & snap_18 & 5.261 & 5.148 \\
\hline snap_19 & 2.407 & 2.342 & snap_19 & 4.965 & 4.880 \\
\hline snap_20 & 2.297 & 2.220 & snap_20 & 4.771 & 4.744 \\
\hline snap_21 & 1.890 & 1.913 & snap_21 & 4.468 & 4.436 \\
\hline snap_22 & 2.337 & 2.379 & snap_22 & 4.925 & 4.929 \\
\hline snap_23 & 2.207 & 2.156 & snap_23 & 4.982 & 4.916 \\
\hline snap_24 & 1.991 & 1.935 & snap_24 & 4.892 & 4.802 \\
\hline snap_25 & 2.253 & 2.251 & snap_25 & 4.637 & 4.577 \\
\hline
\end{tabular}

